\title{
Muon Flux at the Geographical South Pole
}

\author{
X.Bai ${ }^{\text {a,1 }}$ T.K.Gaisser ${ }^{a}$ A.Karle ${ }^{b}$ K.Rawlins ${ }^{\text {b,2 }}$ G.M.Spiczak ${ }^{\text {a,3 }}$ \\ Todor Stanev ${ }^{\mathrm{a}}$ \\ ${ }^{a}$ Bartol Research Institute and Department of Physics and Astronomy, University \\ of Delaware, Newark, DE 19716, USA \\ ${ }^{\mathrm{b}}$ Department of Physics, University of Wisconsin, Madison, WI 53706, USA
}

\begin{abstract}
The muon flux at the South-Pole was measured for five zenith angles, $0^{\circ}, 15^{\circ}, 35^{\circ}$, $82.13^{\circ}$ and $85.15^{\circ}$ with a scintillator muon telescope incorporating ice Cherenkov tank detectors as the absorber. We compare the measurements with other data and with calculations.
\end{abstract}

Key words: muon flux, scintillator detector, ice Cherenkov detector

\section{Introduction and motivation}

The IceCube neutrino telescope [1] under construction at the South Pole includes a surface array, IceTop, for calibration and tagging of cosmic-ray induced background. Together, the surface array and neutrino telescope constitute a three-dimensional air shower array that will also be used to study the cosmic-ray spectrum from $300 \mathrm{TeV}$ to $1 \mathrm{EeV}$ [2]. IceTop consists of ice Cherenkov detectors with two tanks per station near the top of each IceCube string. The surface detector, when complete, will form a kilometer-square air shower array with nearest neighbor spacing between stations of approximately $125 \mathrm{~m}$. The tanks contain clear ice with a surface area of $2.4 \mathrm{~m}^{2}$ and depth of $0.9 \mathrm{~m}$, and each is instrumented with two IceCube digital optical modules

1 Corresponding author. Tel.: (302)831-8165; E-mail address: bai@bartol.udel.edu. 2 present address: University of Alaska Anchorage, 3321 Providence Dr., Anchorage, AK 99508

3 present address: Department of Physics, University of Wisconsin-River Falls, River Falls, WI 54022

Preprint submitted to Elsevier Science $\quad 31$ October 2018 
(DOMs) partially embedded in the ice and facing down. Four two-tank stations with a total of 16 DOMs have been taking data since they were deployed in the 2004-2005 austral summer season [3].

Water Cherenkov detectors were used as the primary detector elements in the Haverah Park surface array [4] and they make up the ground array of the Auger Project in Argentina [5]. Following an early test at the South Pole [6], development of a frozen Cherenkov detector as an element of an air shower array was undertaken in connection with the South Pole Air Shower Experiment (SPASE) [7]. Use of ice tanks as detectors for IceTop requires understanding the background radiation and in particular the muon fluxes at the South Pole. As in the Auger experiment [5], the pulse-charge distribution of single tank hits with its characteristic muon peak will be used for detector calibration and monitoring. In normal data taking muons will not be tagged by a muon telescope, so their fluxes must be well understood as a function of zenith angle on-site. In addition, muons are one of the major components of air showers, becoming especially important for showers at large zenith angle. Therefore detailed study of detector response to muons is needed to design the data acquisition system and interpret waveforms generated by air showers.

Measurements of the muon flux are also of intrinsic interest, for example, in connection with understanding the production spectra of atmospheric neutrinos. Although many muon flux measurements have been made throughout history (see the review by P.K.F Grieder [8]), most of them were done for vertical or nearly vertical muons. Only a few experiments measured nearly horizontal muons above a few GeV, for example at $3220 \mathrm{~m}$ a.s.l [9] and 3250 $m$ a.s.l $[10,11]$. In this work, using the test tanks deployed at the South-Pole as the absorber, we made a controlled and precise measurement of the flux of muons with minimum kinetic energy of several hundred $\mathrm{MeV}$ to about one $\mathrm{GeV}$ and zenith angle from vertical to nearly horizontal at the South Pole ( $\sim 700 \mathrm{~g} / \mathrm{cm}^{2}$, or $\sim 3100 \mathrm{~m}$ a.s.l.). The details of the setup are given in $\S 2$ and the analysis and results of our measurements in $\S 3$. To give a systematic view of the muon flux at this altitude, some data from other measurements are collected in $\S 4$. We conclude in $\S 5$ with a comparison to calculations.

\section{Details of the setup}

Most muons at the surface of the Earth are produced high in the atmosphere from the decay of charged pions generated in the interactions of high energy cosmic rays with atmosphere nuclei. The muon flux varies with altitude and has a strong zenith angle dependence, reflecting the convolution of their production spectrum with their energy loss and decay. At large zenith angles

the muon flux becomes very small, as most of the muons generated in the 
atmosphere decay before reaching the detector. As a consequence, the experimental setups for measuring the nearly vertical and nearly horizontal muon fluxes are different. Near the vertical a simple coincidence requirement is sufficient, whereas the measurement at large zenith angles requires more complex trigger and analysis procedures because of the low event rate.

We show in Fig. 1 the configuration used for measuring the flux of nearly vertical muons. From December 10 to 24, 2000, the integral muon flux was measured at zenith angles of $0^{\circ}, 15^{\circ}$ and $30^{\circ}$. The absorber was a small ice Cherenkov detector installed at the South Pole inside the boundary of the SPASE-2 array [12]. It is a cylindrical polyethylene tank of area of $1.14 \mathrm{~m}^{2}$ and height of $1.24 \mathrm{~m}$. The inside of the tank is lined with white, diffusely-reflecting Tyvek (type 1025D). After filling with the South-Pole station drinking water, two analog AMANDA optical modules (OMs) [13], with a separation of $0.51 \mathrm{~m}$, were mounted facing down symmetrically off-center with their photo cathode region completely submerged. After the tank was frozen, the resulting ice depth was $0.99 \mathrm{~m}$. A muon telescope consisting of three $0.2 \mathrm{~m}^{2}$ scintillators, two stacked on top of the tank and one underneath, identifies penetrating muons by requiring a coincidence among S1, S2 and S3 within 50 ns. Electric pulses from the two OMs and the three scintillators were transmitted through $\sim 100 \mathrm{~m}$ of twisted pair and RG-8 coaxial cables, respectively, to the central SPASE-2 building that houses the electronics and data acquisition system. Three scintillator signals first enter a Phillips 711 discriminator, then go to a Phillips 755 logic unit. Both the singles rate and the coincidence rate were measured by a Jorway 1880B scaler. A 1 GHz Tektronix digital oscilloscope and a Linux PC were used to digitize and read out the waveforms from the two OMs through PCI-GPIB.

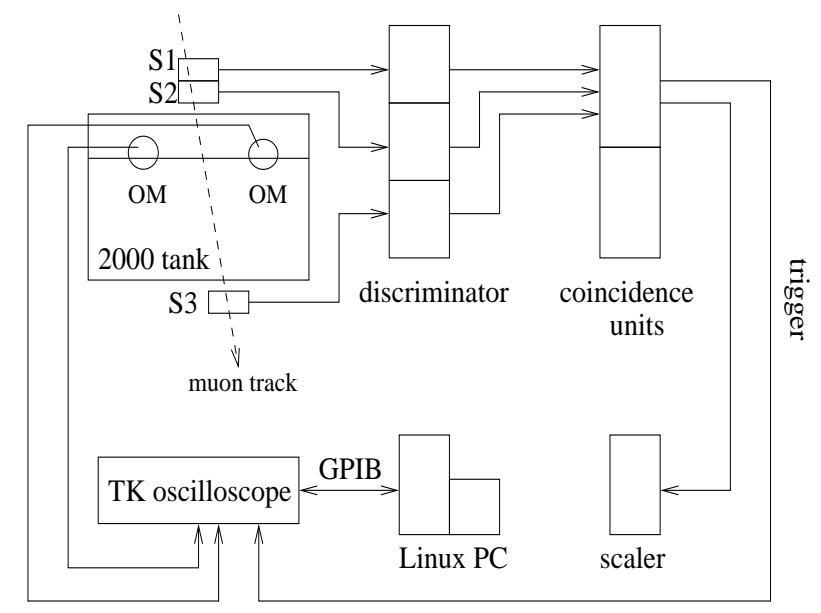

Fig. 1. A sketch of the ice Cherenkov detector (as the absorber), the muon telescope scintillators and the electronics in the flux measurement of $0^{\circ}, 15^{\circ}$ and $30^{\circ}$ muons. Three fold coincidence was used to effectively eliminate the accidental coincidence rate and other background triggers that may form a two fold coincidence by chance. 
The configuration used for the measurement of horizontal muons is shown in Fig. 2. Because of the low rate of horizontal muons the coincidence rate of two scintillators forming a simple muon telescope is dominated by triggers of local small air showers. Reducing the background requires special attention to the detector setup and to the data taking. First, as shown in the setup sketched in Fig. 2, two scintillators marked 'veto-S1' and 'veto-S2' were used in anti-coincidence with $\mathrm{S} 1$ and $\mathrm{S} 2$ respectively. Second, in addition to the waveforms from the two OMs in the tank, the trigger pulse and the traces of the discriminated pulses from the two muon telescope scintillators (S1 and S2 on the figure) were recorded by two digital oscilloscopes to provide the relative timing information among these pulses. The cuts used to filter the data are described in the next section. The singles rate of each scintillator and the coincidence rate with and without anti-coincidence were monitored by a scaler that has a negligible dead time at the working rates. The rates measured by the scalars were then used to correct for the dead time in the signal digitizing and reading system.

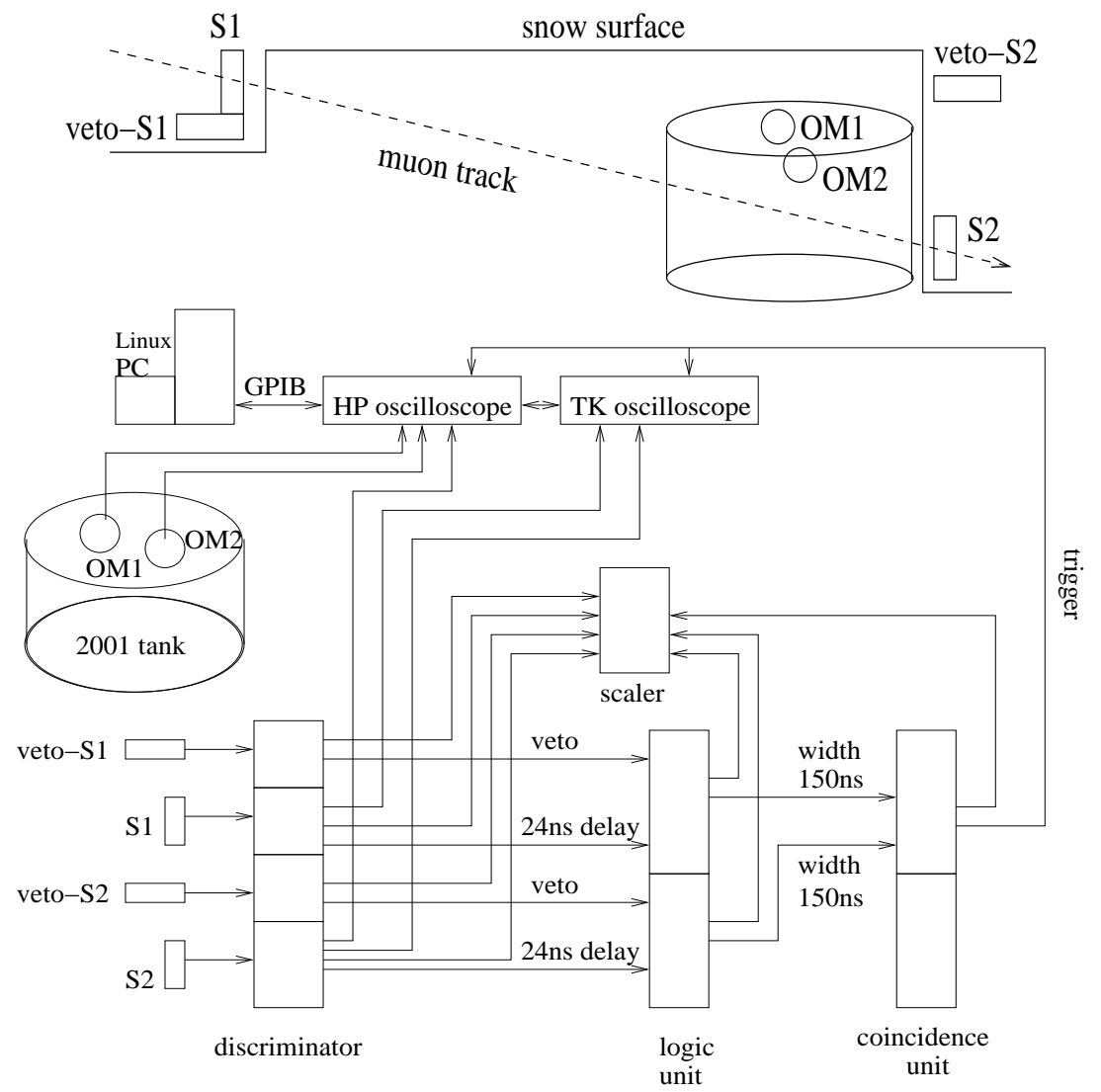

Fig. 2. A sketch of the detector layout (top) and the electronics (bottom) in the flux measurement of $82.13^{\circ}$ and $85.15^{\circ}$ muons. Scintillators $S 1$ and $S 2$ were used to form a muon telescope with veto-S1 and veto-S2 in anti-coincidence.

The data for muon flux measurement at zenith angle $82.13^{\circ}$ and $85.15^{\circ}$ were taken from December 13 to 21 in 2002, during which some time was spent 
to rearrange the cables and detectors in the snow and to survey the detector positions. In this measurement, the tank was a larger cylindrical test tank $\left(3.94 \mathrm{~m}^{2} \times 1.23 \mathrm{~m}\right)$ installed inside the boundary of the SPASE-2 array in 2001. The preparation and installation of this tank were similar to the smaller tank. The two OMs are positioned symmetrically about the center with a separation of $1.07 \mathrm{~m}$. The ice depth in the tank is $1.06 \mathrm{~m}$. Fig. 3 shows a plan view of the coordinates of the tank and scintillators within the SPASE array.

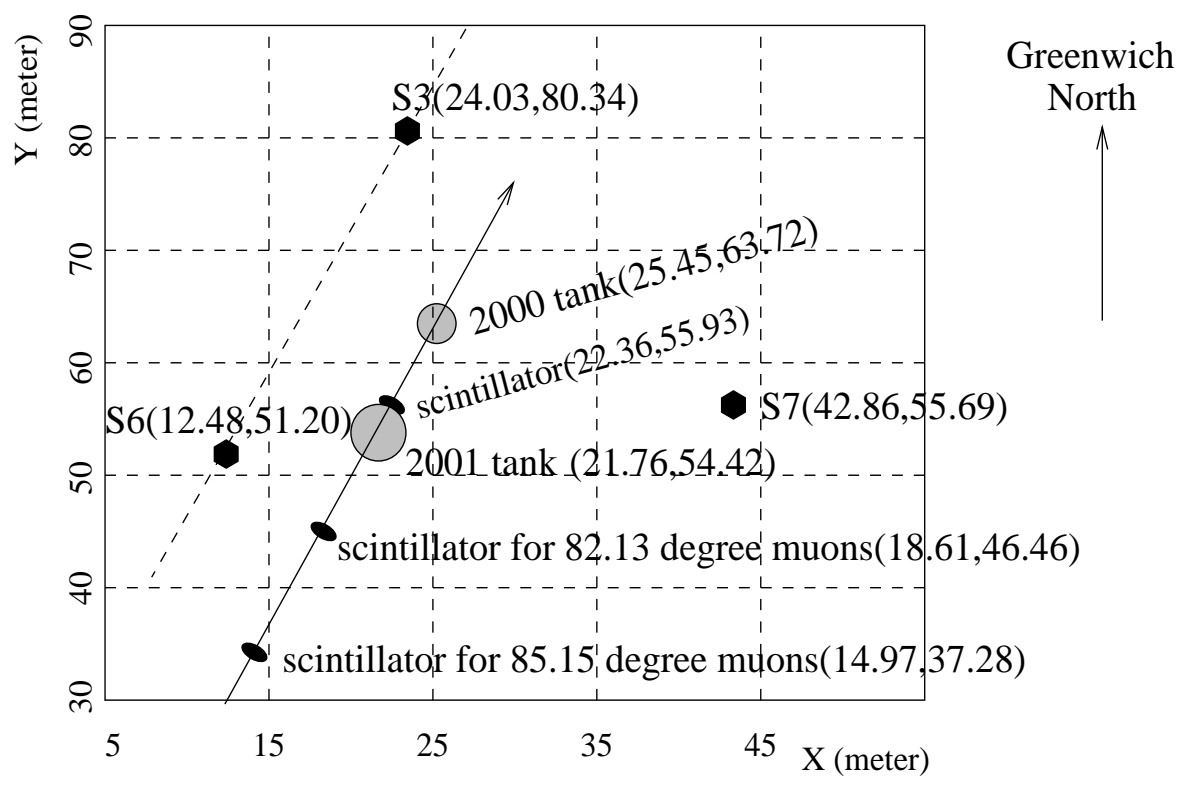

Fig. 3. The coordinates of the detectors in the nearly horizontal muon measurement. S3, S6 and S7 are three of the SPASE-2 stations. Coordinate (X,Y) in meters are given in the SPASE-2 reference frame.

\section{Data analysis and muon flux at the South Pole}

\subsection{Nearly vertical muons}

The flux of penetrating muons at three near vertical zenith angles is summarized in Table 1. Only the statistical errors are given. Column three gives the acceptances of the muon telescope at these three zenith angles. The acceptances were obtained by a Monte Carlo simulation that requires $5.0 \times 10^{4}$ particles to pass through all three scintillators for each case. The last two columns show respectively the integral fluxes and the minimum kinetic energy (and momentum) required for muons to pass through all the three scintillators. The flux is obtained as the measured rate of events divided by the acceptance. 
Table 1

Rates and fluxes of muons near the vertical at the South Pole. The muon threshold kinetic energy was calculated from the table of the continuous-slowing-downapproximation (CSDA) muon range in water [14]. The ice density used in the calculation is $0.92 \mathrm{~g} \cdot \mathrm{cm}^{-2}$.

\begin{tabular}{ccccc}
\hline $\begin{array}{c}\text { zenith }(\min , \max ) \\
\left(^{\circ}\right)\end{array}$ & $\begin{array}{c}\text { rate } \\
(\mathrm{Hz})\end{array}$ & $\begin{array}{c}\text { acceptance } \\
\left(\mathrm{cm}^{2} \cdot \mathrm{sr}\right)\end{array}$ & $\begin{array}{c}\text { integrated flux } \\
\left(\mathrm{cm}^{2} \cdot \mathrm{s} \cdot \mathrm{sr}\right)^{-1}\end{array}$ & $\begin{array}{c}E_{\min }\left(p_{\min }\right) \\
\mathrm{MeV}(\mathrm{MeV} / \mathrm{c})\end{array}$ \\
\hline $0.0(0,16.5)$ & $2.22 \pm 0.01$ & $1.26 \times 10^{2}$ & $(1.76 \pm 0.01) \times 10^{-2}$ & $246.0(335.4)$ \\
$15.0(0.4,28.6)$ & $1.80 \pm 0.01$ & $1.07 \times 10^{2}$ & $(1.68 \pm 0.01) \times 10^{-2}$ & $263.5(353.7)$ \\
$35.0(25.8,44.2)$ & $0.72 \pm 0.01$ & $4.84 \times 10^{1}$ & $(1.49 \pm 0.02) \times 10^{-2}$ & $311.7(403.8)$ \\
\hline
\end{tabular}

\subsection{Nearly horizontal muons}

Because of the low horizontal muon flux and the relatively high rate of local small air showers, the coincidence rate between scintillator $S 1$ and $S 2$ on Fig. 2 is dominated by background even when the two veto scintillators are included in the trigger. Figures 4 and 5 show the distributions of time of flight (TOF) as recorded by scintillators $S 1$ and $S 2,\left(T O F=T_{S 1}-T_{S 2}\right)$, with no cuts (the top histogram) and after various cuts have been applied to the data. The physical distance from $S 1$ to $S 2$ corresponds to a time differences of $T_{S 1}-T_{S 2}=-30.5 \mathrm{~ns}$ for the setup at $82.1^{\circ}$ and to $-63.5 \mathrm{~ns}$ for the larger angle. In the top histogram (without cuts) there is little or no sign of a peak at the expected times. Therefore, simply counting all events on a TOF window would greatly overestimate the true horizontal muon flux. The following series of cuts was applied to reduce the background:

- cut $_{1}$ : For each triggered event, scanning the waveforms recorded by the digital oscilloscopes and choosing those with exactly one hit in the two triggering scintillators and two OMs in a time window of $500 \mathrm{~ns}$.

- cut $_{2}$ : Choosing events in which the peaks of the pulses in the two OMs in the tank are close in time $a b s\left(T_{O M 1}-T_{O M 2}\right) \leq 14 \mathrm{~ns}$. The $14 \mathrm{~ns}$ is related to the slow rise time and the signal fluctuations in the tank. This cut selects those events of which the Cherenkov photons generated in the tank give rise to the signals in the two OMs evenly and simultaneously. The first two cuts are both designed to reduce the number of events caused by showers with several particles spread in time. In addition to single particle events, however, events with a few particles arriving within a time window less than the detector resolving times can still pass these cuts.

- cut $_{3}$ : Choosing events in which abs $\left(T_{O M 1}-T_{S 2}-234 n s\right) \leq 16 n s$. Here, $T_{S 2}$ is the time of the discriminated pulse from the trigger scintillator $S 2$. The average cable delay between the $O M 1$ signal and the signal from scintillator $S 2$ recorded on the oscilloscope is $234 \mathrm{~ns}$. It was determined by a system 
calibration with vertical muons in which we used the same cable, the same trigger threshold, etc. The $16 n s$ is one half of the rise time of the OM pulse, the fluctuation in the tank signal as seen in $O M 1$ and the fluctuation in the signal from scintillator $S 2$. This cut gets rid of those triggers in which the pulse generated in $S 2$, which is located next to the tank, is too far in time from the signal in the tank.

- cut $_{4}$ : Choosing events in which abs $\left(T_{S 1}+T_{T O F}+T_{\text {delay }}\right) \leq \delta(t)$. Here, $T_{S 1}$ is the time (on the oscilloscope trace) of the discriminated pulse from scintillator S1. In the setup, it is a negative number (i.e. to the left of the trigger moment on the oscilloscope trace) for single muon event. $T_{T O F}$ is the muon time-of-flight from S1 to S2, which is $30.5 \mathrm{~ns}$ and $63.5 \mathrm{~ns}$ for muons of $\theta=82.13^{\circ}$ and $\theta=85.15^{\circ}$ in this setup. $T_{\text {delay }}$ is the $50 \mathrm{~ns}$ trigger delay set in the digital oscilloscope. For single muon events, abs $\left(T_{S 1}+T_{T O F}+T_{\text {delay }}\right)$ is the time jitter relative to the DAQ trigger moment which is $0 \mathrm{~ns}$ on the oscilloscope trace. In the analysis, $\delta(t)$ was chosen $4.5 \mathrm{~ns}$, which corresponds to the time jitter in the scintillator and the electronics.
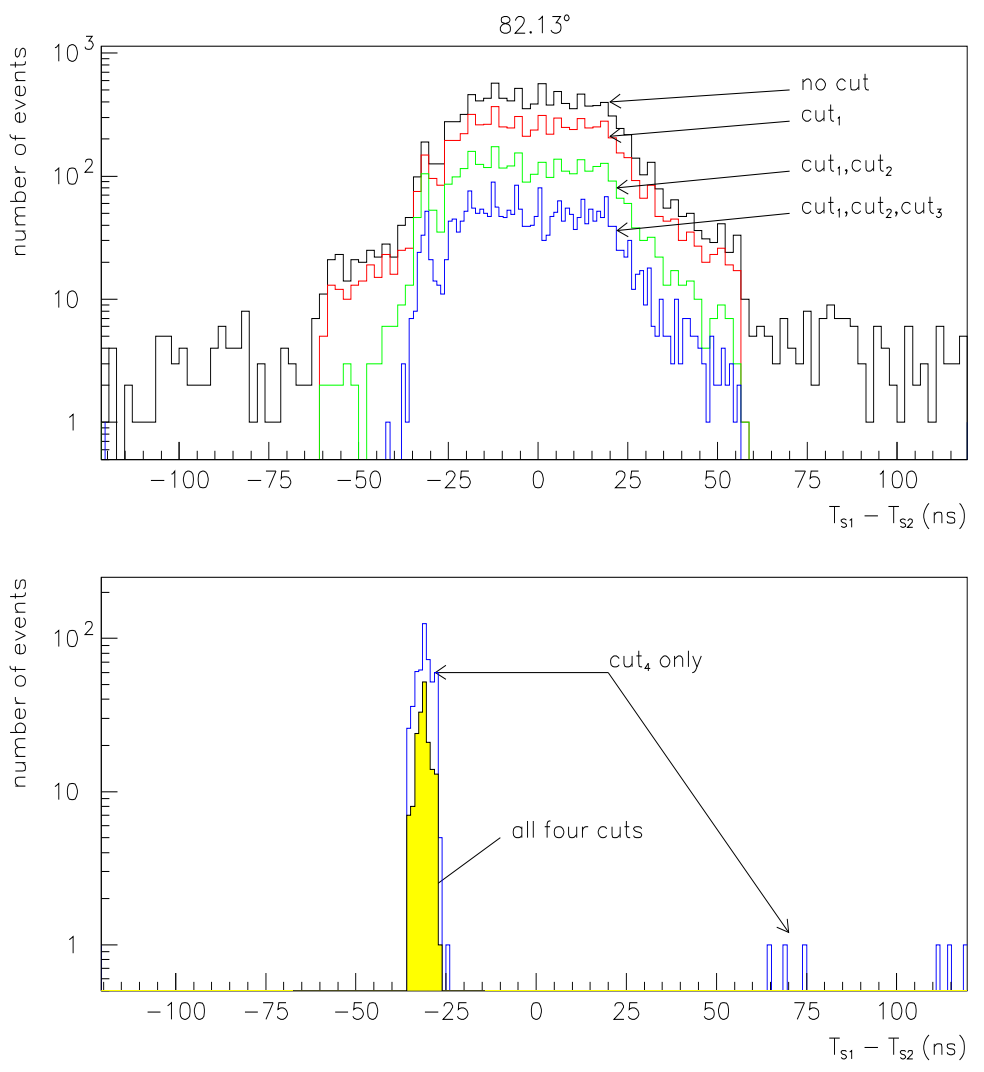

Fig. 4. The TOF $\left(T_{S 1}-T_{S 2}\right)$ spectra of triggered events in $\theta=82.13^{\circ}$ muon measurement. The cuts used for selecting the events under each histogram are marked. See more details about the cuts in the text. 

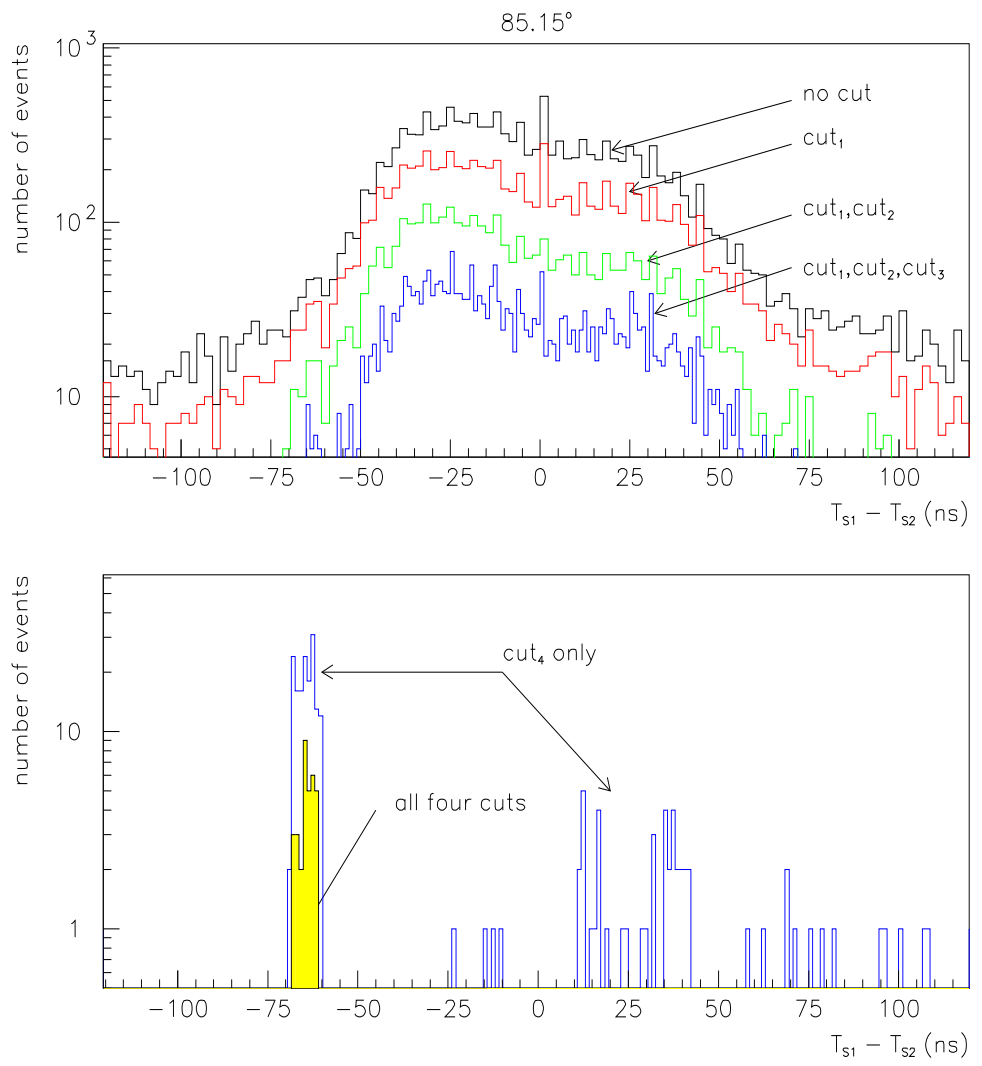

Fig. 5. The TOF spectrum of triggered events in $\theta=85.15^{\circ}$ muons measurement.

In Fig. 4 and 5, from top to bottom, the $S 1$ to $S 2$ TOF spectrum are shown for triggered events without any cut, and events left after the application of cuts 1 to 4 . The following features on the TOF spectra are noted:

(a) The events left after the application of the first three cuts start to show a clear peak at the correct TOF position on the spectrum although none of these cuts makes use of the time-of-flight from $S 1$ to $S 2$;

(b) Although most of the events that survive the cut $_{4}$ (and no other cuts) distribute themselves around the expected muon flight time on the TOF spectrum, several of them still fall outside the expected time window in Fig. 4, and more in Fig. 5;

(c) All the events left after all four cuts show themselves at the expected position as on an ideal TOF spectrum of single muon events;

(d) The events in the peak with all four cuts applied correspond to those events that make up the peak on the distribution with cuts 1,2 and 3 .

We therefore conclude that the events left after application of all four cuts are true horizontal muon events. It is interesting to compare their waveforms with 
vertical muon waveforms as seen by the two OMs in the same tank. We note two features in the data:

(1) The average amplitude of these pulses is about 2 times as big as that of the vertical muons.

(2) The average rise time of the these pulses in the tank is 3ns longer than that of vertical muon pulses. The fall time of the average waveform is nearly the same as that of the average waveform of vertical muons.

A GEANT-4 simulation of the tank response was carried out for both vertical muons and muons of zenith angle $82.13^{\circ}$ and $85.15^{\circ}$ [15]. The two features listed above are well reproduced in the simulation. They are directly related to the longer track length of the near horizontal muons in the tank.

Taking the events left after all four cuts as the single muon events, we summarize the measurement in Table 2.

Table 2

Summary of the horizontal muon measurement at the South-Pole. The data taking time excludes dead time. The systematic errors are from the uncertainty in the cut parameters. They were obtained by a systematic comparison of the S1 to S2 time difference spectra under four cuts with different parameter values. The muon threshold kinetic energy was calculated from the table of the continuous- slowingdown-approximation (CSDA) muon range in water [14]. The ice and the snow densities used in the calculation are $0.92 \mathrm{~g} \cdot \mathrm{cm}^{-2}$ and $0.4 \mathrm{~g} \cdot \mathrm{cm}^{-2}$. The latter has an error of $\pm 5 \%$.

\begin{tabular}{ccc}
\hline zenith coverage $\left(^{\circ}\right)$ & $82.13 \pm 2.97$ & $85.15 \pm 1.44$ \\
time-of-flight $(n s)$ & 30.5 & 63.5 \\
number of events & 173 & 33 \\
acceptance $\left(\mathrm{cm}^{2} \cdot \mathrm{sr}\right)$ & 4.6881 & 1.0944 \\
data taking time $(\mathrm{sec})$ & $2.1227 \times 10^{5}$ & $2.5600 \times 10^{5}$ \\
$E_{\text {min }}(\mathrm{MeV})\left(p_{\min }(\mathrm{MeV} / \mathrm{c})\right)$ & $899 \pm 45(999 \pm 50)$ & $1752 \pm 87(1855 \pm 93)$ \\
statistical errors & $\pm 7.6 \%$ & $\pm 17.4 \%$ \\
systematical errors & ${ }^{+14 \%}$ & ${ }_{-12 \%}^{3 \%}$ \\
flux $\left(\mathrm{cm}^{2} \cdot s \cdot s r\right)^{-1}$ & $\left(1.74_{-0.22}^{+0.24} \pm 0.13\right) \times 10^{-4}$ & $\left(1.18_{-0.14}^{+0.04} \pm 0.21\right) \times 10^{-4}$ \\
\hline
\end{tabular}




\section{Muon fluxes by some other experiments at an altitude close to the South-Pole}

There are several measurements of the vertical muon flux at high altitude that are performed at different muon threshold energies, geomagnetic locations and solar epochs. At $3220 \mathrm{~m}$ a.s.l, the vertical intensity of muons above $2 \mathrm{GeV}$ was measured with GM hodoscope by Shen and Chiang [9]. The reported flux is $(4.9 \pm 0.2) \times 10^{-3}\left(\mathrm{~cm}^{2} \cdot s \cdot \mathrm{sr}\right)^{-1}$. For muons of energy $\geq 162.9 \mathrm{MeV}$, the vertical integral intensity at the South-Pole altitude is $(2.61 \pm 0.01) \times 10^{-2}\left(\mathrm{~cm}^{2} \cdot \mathrm{s} \cdot \mathrm{sr}\right)^{-1}$ according to the curve on figure 2.130 in reference [8], which was made from the measurement by Blokh et al. [16]. Vertical muons at $2960 \mathrm{~m}$ a.s.l. and $3250 \mathrm{~m}$ a.s.l. were measured by Allkofer and Trümper [17] and Kocharian et al. [18]. By integrating the differential momentum spectrum on figure 2.137 in reference [8], the integral muon intensities were found to be $I_{2960 \mathrm{~m}}(\geq 203 \mathrm{MeV} / \mathrm{c})=1.16 \times 10^{-2}\left(\mathrm{~cm}^{2} \cdot \mathrm{s} \cdot \mathrm{sr}\right)^{-1}$ and $I_{3250 m}(\geq 414 \mathrm{MeV} / \mathrm{c})=1.58 \times 10^{-2}\left(\mathrm{~cm}^{2} \cdot \mathrm{s} \cdot \mathrm{sr}\right)^{-1}$. Some of the differences between these results can be explained by the different altitudes and muon threshold energies. The difference in altitude between Ref. [17] and South Pole corresponds to about $30 \mathrm{~g} / \mathrm{cm}^{2}$, which increases the energy threshold by 60 $\mathrm{MeV}$. In addition, there is also a decrease of the muon flux because of muon decay. About $15 \%$ of the muons at threshold decay in $300 \mathrm{~m}$. These effects, as well as the solar epochs, do not explain all the differences among the quoted measurements. Our result is in the middle of the scattered points obtained by those previous work and agrees the best with the result of Ref. [18].

Few experiments have been done for low energy nearly horizontal muons with a small zenith bin. For an angular interval from $78.4^{\circ}$ to $90^{\circ}$, corresponding to a mean of $86.2^{\circ}$, the intensity at $3220 \mathrm{~m}$ a.s.l was reported to be [9]: $I_{h}(\geq$ $2 \mathrm{GeV})=(9.68 \pm 0.32) \times 10^{-5}\left(\mathrm{~cm}^{2} \cdot s \cdot s r\right)^{-1}$. Because of the large angular range and the steep dependence of the muon flux on the cosine of the zenith angle this number which is lower than the current measurement is difficult to interpret.

Asatiani et al. [10] carried out a spectral measurement at $3250 \mathrm{~m}$ a.s.l with a large magnetic spectrometer. The muon differential spectrum covers the range from $10 \mathrm{GeV} / \mathrm{c}$ to $2000 \mathrm{GeV} / \mathrm{c}$. The muon incident angle is from $80^{\circ}$ to $90^{\circ}$ with the mean zenith angle $84^{\circ}$. The presented spectrum was normalized to the calculation by Ashton et al. [19] at $50 \mathrm{GeV}$ for the zenith angle $84^{\circ}$. Integrating over the differential energy spectrum in figure 2.139 in reference [8] gives an approximate intensity of $(8.85 \pm 0.02) \times 10^{-5}\left(\mathrm{~cm}^{2} \cdot \mathrm{s} \cdot \mathrm{sr}\right)^{-1}$.

With the same instrument, Asatiani et al. [11] reported the differential muon spectra at narrow zenith angle intervals covering from $80^{\circ}$ to $88^{\circ}$. The integral muon flux in units of $\left(\mathrm{cm}^{2} \cdot \mathrm{s} \cdot \mathrm{sr}\right)^{-1}$ above a given energy threshold for four 
zenith bins are: $80^{\circ}-82^{\circ}, I_{>16.5 \mathrm{GeV}}=1.27 \times 10^{-4} ; 82^{\circ}-84^{\circ}, I_{>16.5 \mathrm{GeV}}=$ $1.01 \times 10^{-4} ; 84^{\circ}-86^{\circ}, I_{>25.8 \mathrm{GeV}}=5.60 \times 10^{-5} ; 86^{\circ}-88^{\circ}, I_{>14.8 \mathrm{GeV}}=4.40 \times 10^{-5}$.

Because of the finer binning in the zenith angle we can correct these numbers for the difference in muon threshold and compare to the current measurement. The correction was done using the calculation of Ref. [20] for the fluxes of muons at $\cos \theta=0.25,0.15$ and 0.05 at the sea level. The muon fluxes from this calculation were interpolated for the average $\cos \theta$ and energy threshold in the measurement and compared to the fluxes above $2 \mathrm{GeV}$ energy threshold. The scaling factors for the four points were found to be 1.84, 1.60, 1.77 and 1.22 respectively. The data of Ref. [11], after being scaled to muon energy threshold of $2 \mathrm{GeV}$, are shown with open squares in Fig. 6. The agreement is excellent.

\section{Comparison with simulation}

The measurements were compared with simulations. For the vertical and near vertical muons, a Monte Carlo simulation was carried out to calculate the fluxes expected for the average atmosphere depth of $702 \mathrm{~g} / \mathrm{cm}^{2}$ during the muon measurement. The simulation used TARGET2.1 [21] and the cosmic ray flux of reference [20] for the epoch of solar maximum. The muon fluxes of $1.80 \times 10^{-2}, 1.73 \times 10^{-2}$ and $1.68 \times 10^{-2}\left(\mathrm{~cm}^{2} \cdot \mathrm{s} \cdot \mathrm{sr}\right)^{-1}$ were predicted for the three angles. The measured muon fluxes at $0^{\circ}$ and $15^{\circ}$ are in excellent agreement with the prediction, while at $35^{\circ}$ the flux is lower by $12 \%$.

The fluxes of nearly horizontal muons by this work are compared to the the simulation of Ref. [20]. The current measurements are shown with solid squares in Fig. 6. The predicted angular dependence of muons with energy above 1 (1.86) GeV from Ref. [20] are shown with a dashed (solid) line. One can see that the expected fluxes for the two muon energy thresholds at $\cos \theta$ less than 0.2 become the same. This is because the horizontal muon energy spectrum has very few muons in this energy range. The good agreement between the measurements and the calculations also confirms that accounting for difference in the altitude of the measurements is not important for nearly horizontal muons.

\section{Summary}

Using the prototype IceTop ice Cherenkov detector as the absorber, data has been collected for muons in five directions from vertical and nearly vertical to nearly horizontal. To select the rare nearly horizontal muon events, four cuts 


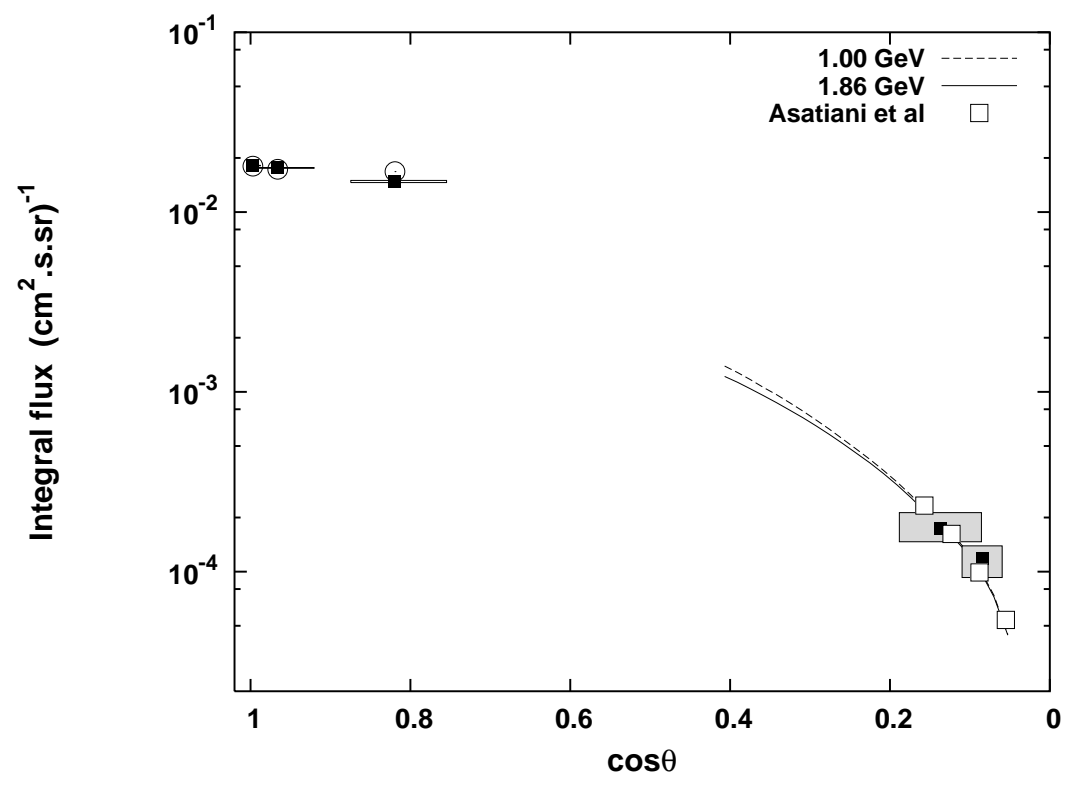

Fig. 6. The measured integral flux of muons above the energy thresholds specified in Tables 1 and 2 are shown with full squares versus the cosine of the zenith angle. The shaded boxes around data points show the errors in the fluxes and the zenith angular coverage in the measurement. The open circles show the calculation for vertical and near vertical muons at the South Pole. The lines show the angular dependence of the predicted integral fluxes above the two energy thresholds. The open squares are the measurements of Ref. [11] after being scaled to a threshold of $2 \mathrm{GeV}$.

were developed in the data analysis. The procedure for selecting horizontal muons was crosschecked by a GEANT-4 simulation of the muon waveforms seen in the OMs.

The integral muon flux of muons at $0^{\circ}$ and $15^{\circ}$ agrees very well with the simulation, which accounts for the atmospheric depth and the specific atmospheric density profile above the South Pole. The simulation of Ref. [20] is not done for the South Pole location, however this is not important for the high energy muons that survive at very large zenith angles.

\section{Acknowledgements}

The work was supported by the Office of Polar Programs of the U.S. National Science Foundation under grants OPP-998081 and OPP-0236449. The authors gratefully acknowledge the support from the U.S. Amundsen-Scott South Pole station. The assistance from Jerry Poirier during the detector deployment in 2000 and 2001, the GEANT-4 simulation done by Ralf Ulrich, and the acceptance calculation in the near vertical muon measurement by Zhe Ma are 
highly appreciated as well.

\section{References}

[1] J. Ahrens et al. Astropart. Phys. 20, 507(2003). See also Icecube Project Preliminary Design Document, http://icecube.wisc.edu/pub_and_doc/90000010.curr.pdf

[2] T.K. Gaisser for the IceCube Collaboration, "IceTop: the Surface Component of IceCube", Proc. $28^{\text {th }}$ Int.Cos.Ray Conf., Tsukuba, Japan, HE1.5, 1117(2003) and also T. Stanev, "IceTop Status in 2004", Nucl. Phys. B (Proc. Suppl) 145, $327(2005)$

[3] T.K. Gaisser for the IceCube Collaboration, "Air showers with IceCube: First Engineering Data" Proc. $29^{\text {th }}$ Int.Cos.Ray Conf., 2005, Pune, India. See also astro-ph/0509330, pp60-63.

[4] M. Ave, J. Knapp, J. Lloyd-Evans, M. Marchesini, \& A.A. Watson, Astropart. Phys. 19, 47(2003)

[5] Auger Collaboration (S. Ranchon, M. Urban), NIM-A538, 483(2005). See also Auger Collaboration, J. Abraham et al., NIM-A523, 50(2004)

[6] S.Barwick and J.Beaman, Proc. $23^{\text {rd }}$ Int.Cos.Ray Conf., Calgary, 4, 683(1993)

[7] X.Bai et al., Proc. $27^{\text {th }}$ Int.Cos.Ray Conf., Hamburg, Germany, 3, 981(2001)

[8] Peter K.F.Grieder, "Cosmic Rays at Earth Researcher's Reference Manual and Data Book", Elsevier, 2001.

[9] Shen Chang-quan and Chiang In-lin $16^{\text {th }}$ Int.Cos.Ray Conf., Kyoto, Japan, 10, $8(1979)$

[10] T.L.Asatiani et al. $14^{\text {th }}$ Int.Cos.Ray Conf., Munchen, Germany, 6, 2024(1975)

[11] T.L.Asatiani et al. $18^{\text {th }}$ Int.Cos.Ray Conf., Bangalore, India, 7, 47(1983)

[12] J.E.Dickinson et al. NIM-A 440, 95(2000)

[13] E.Andres et al. Nature 410, 441(2001)

[14] D.E.Groom, et al. http ://panisse.lbl.gov/ deg/muon.html

[15] Ralf Ulrich private communication, 2003

[16] Y.L.Blokh, et al. Nuovo Cimento 37B, 198(1977)

[17] Allkofer, O.C., and J. Trümper, Z. Naturforsch 19A, 1304(1964)

[18] Kocharian, N.M. et al. Soviet Phys. JETP 3, 350(1956)

[19] F.Ashton et al. Proc.Phys.Soc. 87, 79(1966) 
[20] Vivek Agrawal, T.K. Gaisser, Paolo Lipari, and Todor Stanev Phys. Rev. D53, 1314(1996)

[21] R. Engel, T.K. Gaisser, P. Lipari, and T. Stanev, Proc. $27^{\text {th }}$ Int.Cos.Ray Conf., Hamburg, Germany. 4, 1381(2001) 